\title{
Pregnancy in Post-splenectomized Myasthenia Gravis: A Case Report
}

\author{
Nilofar Noor ${ }^{1}$, Vidushi Kulshrestha ${ }^{2}$, Kallol Kumar Roy ${ }^{3}$, Kandala Aparna Sharma ${ }^{4}$
}

\begin{abstract}
Aim and objective: We aim to emphasize the principles of management of pregnant women with a complex medical condition such as myasthenia gravis.

Background: Myasthenia gravis is an autoimmune disorder affecting neuromuscular transmission resulting in fatigable weakness of skeletal muscles.

Case description: We report a case of pregnancy with myasthenia gravis with a history of splenectomy. The patient was on oral pyridostigmine and prednisolone, which were continued in pregnancy. The patient received multidisciplinary care involving obstetrician, neurologist, anesthetist, and had an uneventful antenatal course. She had a normal vaginal delivery and her postpartum period was uneventful with neonate not developing any signs of myasthenia.

Conclusion: This case highlights the importance of a multidisciplinary approach in managing pregnancy with myasthenia gravis for optimum maternal and neonatal outcomes. The patient should continue the drugs for myasthenia throughout pregnancy. Vaginal delivery is not contraindicated, with preparedness for intubation to tackle myasthenia crisis. Instrumental delivery may be needed for inadequate bearingdown efforts. Neonates should be monitored for $\mathbf{4 8}$ hours for transient neonatal myasthenia symptoms and may need pyridostigmine till symptoms resolve.

Clinical significance: Autoimmune conditions like myasthenia gravis are not uncommon in women in the reproductive age-group. Hence, obstetricians need to acquaint themselves with the knowledge to ensure early diagnosis and appropriate management for a safe pregnancy outcome.

Keywords: Myasthenia crisis, Myasthenia gravis, Pregnancy, Pyridostigmine.

Journal of Postgraduate Medicine, Education and Research (2021): 10.5005/jp-journals-10028-1443
\end{abstract}

\section{BACKGROUND}

Myasthenia gravis is an acquired autoimmune neuromuscular disease where antibodies are present against acetylcholine receptors on the neuromuscular endplates of skeletal muscles. It is characterized by fatigable weakness of the diaphragm, ocular, oropharyngeal, and limb muscles. Symptoms include progressive weakness worsening over the day, ptosis, dysphagia, and dyspnea. ${ }^{1}$ Prevalence is around 50 to 125 cases per million worldwide. Incidence is twice as common in women particularly in the second and third decades of life. ${ }^{2}$ Since this is the reproductive age-group, obstetricians need to be acquainted with its management. In this case report, we aim to summarize the interdisciplinary diagnostic and therapeutic strategies for medical management of myasthenia in pregnancy, puerperium.

\section{Case Description}

A 38-year-old, primigravida, a known case of myasthenia gravis for seven years, was referred from a neurology clinic at 22 weeks of gestation. She underwent splenectomy 5 years back and her symptoms were controlled on oral pyridostigmine $60 \mathrm{mg}$ 8 hourly and oral prednisolone $20 \mathrm{mg}$ daily. A multidisciplinary team including an obstetrician, neurologist, anesthetist, and neonatologist provided care throughout her pregnancy with regular antenatal visits, fetal surveillance, and monitoring for worsening of myasthenia symptoms.

The patient's antenatal course was uneventful. She was admitted at 38 weeks in spontaneous labor. The anesthesiology team, neonatology unit, and the neurologist were immediately
${ }^{1-4}$ Department of Obstetrics and Gynaecology, All India Institute of Medical Sciences, New Delhi, India

Corresponding Author:Vidushi Kulshrestha, Department of Obstetrics and Gynaecology, All India Institute of Medical Sciences, New Delhi, India, Phone: +91 9891910880, e-mail: drvidushi.kul@gmail.com

How to cite this article: Noor N, Kulshrestha V, Roy KK, et al. Pregnancy in Post-splenectomized Myasthenia Gravis: A Case Report. J Postgrad Med Edu Res 2021;55(4):183-185.

Source of support: Nil

Conflict of interest: None

intimated. Intravenous $100 \mathrm{mg}$ stress dose of hydrocortisone was given as the patient had been on long-term oral steroids. The patient was closely watched for any signs of myasthenia crisis and anesthetists prepared for intubation should the need arise. Progress of labor was satisfactory without requiring augmentation. Maternal bearing-down efforts were adequate and she delivered a $2.7 \mathrm{~kg}$ female baby with an Apgar score of $7 / 8$.

In the postpartum period, close monitoring for myasthenia crisis was continued. Neonate was observed for 48 hours and did not develop any signs of congenital myasthenia. Mother and baby were discharged in a healthy state on the third postpartum day.

\section{Discussion}

Pregnancy has a variable effect on the clinical course of myasthenia gravis; with improvement seen in $20-40 \%$ cases; or worsening seen

(c) The Author(s). 2021 Open Access This article is distributed under the terms of the Creative Commons Attribution 4.0 International License (https://creativecommons. org/licenses/by-nc/4.0/), which permits unrestricted use, distribution, and non-commercial reproduction in any medium, provided you give appropriate credit to the original author(s) and the source, provide a link to the Creative Commons license, and indicate if changes were made. The Creative Commons Public Domain Dedication waiver (http://creativecommons.org/publicdomain/zero/1.0/) applies to the data made available in this article, unless otherwise stated. 
in about $14-19 \%$ cases during pregnancy and $16-28 \%$ patients within 6 weeks postpartum. Approximately 40\% of women have an unchanged disease course, ${ }^{1}$ as was seen in our case.

Myasthenia-associated risk of mortality is highest in the first year following the onset of disease and is lowest after 7 years. ${ }^{1}$ Hence, newly diagnosed cases should be counseled to avoid pregnancy. Maternal complications during pregnancy include prolonged labor, myasthenic crisis, and anesthesia-related risks if a cesarean is required. Myasthenia crisis is a potentially lifethreatening exacerbation of the disease which may complicate up to $20 \%$ of cases. ${ }^{1}$ During the crisis, mechanical ventilation and ICU care may be needed for severe respiratory weakness. Known triggers are infection, surgery, general anesthesia, hypo- or hyperthyroidism, physical or emotional stress, extreme temperatures, and medications that may increase muscle weakness. ${ }^{1}$ Pregnancy and postpartum state are independent factors for exacerbation. ${ }^{1}$

The first-line treatment option for myasthenia remains medical; pyridostigmine, prednisolone, and azathioprine are commonly used drugs for symptom control in pregnancy and postpartum and are considered safe. However, very high doses of pyridostigmine are known to induce premature uterine contractions and preterm labor, prednisolone is associated with fetal growth restriction and azathioprine may cause maternal and fetal bone marrow suppression. ${ }^{1,3} \mathrm{IVIg}$ and plasma exchange are reserved to tide over myasthenia crisis. ${ }^{1,3}$ Fortunately, our patient did not exhibit any adverse side effects of the drugs.

Patients not responding to medical treatment may require thymectomy which is reported to improve symptoms in nearly $80 \%$ of myasthenia patients. Since the effect may take years to become apparent, ${ }^{1}$ it is usually performed before 50 years of age. Thymectomy is avoided during pregnancy and, if required, should be performed either preconceptionally or post-delivery.

Splenectomy is also one of the treatment options for managing refractory myasthenia as reported by few authors. Kuzin et al. ${ }^{4}$ discussed the results of splenectomy carried out on 20 patients with generalized myasthenia in whom previous complex treatment including thymectomy, corticosteroid, and azathioprine therapy, and repeated courses of plasmapheresis did not benefit. There was an improvement in $65 \%$ of patients as evidenced by myography and by the reduction in required doses of corticosteroids and anticholinesterase agents. He suggested the inclusion of splenectomy into the multimodality treatment of severe myasthenic patients resistant to standard therapy. ${ }^{4}$ Splenectomy alleviates the disease by reducing the number of lymphocytes as well as the reducing total bulk of the immune system in autoimmune disease. ${ }^{5}$ Limited data are available for the pregnancy outcome in the splenectomized myasthenic women as thymectomy has now become the mainstay surgical option.

Pregnant women with myasthenia should follow at a tertiary center, with facilities of ICU, NICU, and multidisciplinary care involving obstetrician, anesthesiologist, neonatologist, and neurologist. They should be screened for gestational diabetes and fetal growth restriction especially if on steroid therapy. Any infection should be treated promptly which might be a potential trigger for the crisis. Antibiotics such as fluoroquinolones, macrolides, and aminoglycosides should be avoided as these may exacerbate the disease. ${ }^{1,3}$

Managing preeclampsia can be particularly challenging in myasthenic patients. Magnesium sulfate, $\beta$-blockers, and calcium channel blockers can potentially worsen myasthenic symptoms and should be avoided. ${ }^{1,6}$ Levetiracetam and valproic acids are acceptable alternatives for seizure prophylaxis. Methyldopa and oral hydralazine can be used as the initial antihypertensives of choice, while intravenous hydralazine can be used to reduce acute blood pressure in severe hypertension. ${ }^{6}$

Vaginal delivery is preferable in myasthenia gravis and cesarean section is reserved for obstetric indications, bearing in mind the risk of exacerbation during the stress of surgery. ${ }^{1}$ Smooth muscles are spared by the disease, ${ }^{1}$ which explains unaffected uterine contractions and the first stage of labor in our patient. Maternal bearing-down efforts in the second stage of labor may result in fatigue of voluntary striated abdominal muscles causing exhaustion and prolonged second stage. ${ }^{1}$ This may necessitate instrumental vaginal delivery to cut short the second stage. ${ }^{1}$ However, our patient neither had a prolonged second stage nor required instrumental delivery. This might be credited to her well-controlled disease status before parturition.

Adequate analgesia during labor and puerperium is vital to prevent the myasthenic crisis. Epidural anesthesia with amidetype local anesthetic agents is preferred in both vaginal and cesarean deliveries. ${ }^{1}$ General anesthesia is avoided due to the risk of myasthenia crisis. ${ }^{1}$ Non-steroidal anti-inflammatory drugs and paracetamol can be used for postpartum or postoperative pain, while narcotic analgesic agents that may cause respiratory depression should be avoided. ${ }^{1}$

Breastfeeding is not contraindicated and women should be observed for signs of infection or exacerbation., ${ }^{1,3}$ Transplacental passage of maternal antibodies may cause transient neonatal myasthenia in 10-20\% of cases. ${ }^{1}$ Neonate is observed for at least 48 hours as symptoms commonly occur in 12-48 hours and resolve in 4 weeks. ${ }^{1}$ Symptomatic neonates can be treated with pyridostigmine, ventilation, and plasma exchange in severe cases. ${ }^{1}$ Arthrogryposis multiplex congenital, a rare complication with joint contractures is also reported in a neonate due to reduced fetal movements in utero. ${ }^{1,3}$

Multidisciplinary care with adequate control of disease and regular monitoring led to an optimum pregnancy outcome in our case with no complications. As a part of labor preparations, the team was prepared to tackle the myasthenia crisis, keeping the intubation trolley ready.

\section{Conclusion}

Multidisciplinary care with adequate control of disease before and during pregnancy, regular monitoring, and preparedness for potential complications are paramount for an optimum maternal and neonatal outcome in pregnancy with myasthenia gravis. Though nowadays thymectomy is the preferred surgical option, a preconceptional splenectomy can also be considered in severe cases for achieving symptom control and favorable pregnancy outcome.

\section{Clinical Significance}

Autoimmune conditions like myasthenia gravis are not uncommon in women in the reproductive age-group. Hence, obstetricians need to acquaint themselves with the knowledge to ensure early diagnosis and appropriate management for a safe pregnancy outcome. 


\section{Ethical and Humane Considerations}

All procedures performed in the study were in accordance with the ethical standards of the 1964 Helsinki declaration and its later amendments or comparable ethical standards.

\section{Informed Consent}

Informed written consent has been obtained from the patient for the publication of the case report. The patient understands that her name and initials will not be published and due efforts will be made to conceal her identity.

\section{References}

1. Hassan A, Yasawy ZM. Myasthaenia gravis. Sultan Qaboos Univ Med J 2017;17(3):e259-e267. DOI: 10.18295/squmj.2017.17.03.002.
2. Mantegazza R, Baggi F, Antozzi C, et al. Myasthenia gravis (MG): epidemiological data and prognostic factors. Ann N Y Acad Sci 2003;998(1):413-423. DOI: 10.1196/annals.1254.054.

3. Norwood F, Dhanjal M, Hill M, et al. Myasthenia in pregnancy: best practice guidelines from a UK multispecialty working group. J Neurol Neurosurg Psychiatry 2014;85(5):538-543. DOI: 10.1136/jnnp-2013305572.

4. Kuzin MI, Shkrob OS, Gekht BM, et al. Splenectomy in the treatment of severe generalized myasthenia gravis (first experience). Khirurgiia (Sofiia) 1990;8(8):95-99.

5. Hofmann WE, Reuther P, Schalke B, et al. Splenectomy in myasthenia gravis: a therapeutic concept? J Neurol 1985;232(4):215-218. DOI: 10.1007/BF00313782.

6. Ozcan J, Balson IF, Dennis AT. New diagnosis myasthenia gravis and preeclampsia in late pregnancy. BMJ Case Rep 2015;2015(feb26 1):bcr2014208323. DOI: 10.1136/bcr-2014-208323. 